\title{
DISCUSSION AFTER PAPER BY MUSTEL
}

Tayler: I wish to provoke some discussion between observers and theoreticians. Theoreticians require supernovae to produce large amounts of heavy elements and that very large mass loss should occur. However, it is not clear that observers ever see a very high loss of mass or very large amounts of heavy elements. Is this a serious discrepancy?

Mustel: It is very difficult to detect heavy elements from the analysis of the spectra of the envelopes ejected by supernovae. In fact the abundance of all elements of this group is usually very small. Therefore the number of absorbing atoms of any heavy element in the envelope $\left(\mathrm{cm}^{-2}\right)$ is also small. And due to the very large velocity gradient inside the envelopes the absorption lines of these elements are expected to be very shallow. Thus their detection in the spectra of supernovae with a lot of strong blends is very difficult.

Nevertheless it is interesting to point out that the line of BaII $5854 \AA$ in the spectra of type I supernova 1966j in NGC 3198 was anomalously strong, see Mustel (1974). It is very important to continue the efforts to detect similar lines in the spectra of other supernovae.

Arnett: What is the typical star, which becomes a supernova? Suppose stars more massive than $8 M_{\odot}$ do so. The typical one by number would be about $15 M_{\odot}$, while by mass it would be about twice as massive. The nucleosynthesis involves a mass average; observations of supernovae are on averaging by number. Some differences in the objects are to be expected.

Another problem is that the observer of a supernova explosion sees only the outer parts of the exploding object, only a study of remnants will tell us about the rest of the matter.

Finally take the $15 M_{\odot}$ model which I showed earlier. If it loses its envelope and a $4 M_{\odot}$ helium core is left, then an object like the Crab nebula should result: a neutron star and a helium rich nebula of about $2 M_{\odot}$.

Audouze: The discrepancy between observations of supernovae, where only light elements are seen, and the theories, where heavy elements are supposed to be produced in large amounts, is only apparent. There is now a rather large consensus that supernovae are the sources of cosmic rays, this is substantiated in particular by energy arguments. In the cosmic rays the heavy elements are observed in larger amounts than in the so-called universal abundances. It seems then that there is experimental evidence that supernovae produce heavy elements in the large amount predicted by theorists.

Tayler: Although the connection between supernovae and cosmic rays is probably valid, this is in reality only assuming one thing which you think plausible in order to prove another. 
McCarthy: Speaking about possible observational evidence of heavy elements may we point out that the $\mathrm{S}$ stars mentioned by Miss Sackmann as possible forerunners of novae and supernovae show technetium as Paul Merrill and Mrs Sitterly have pointed out. Perhaps studies at higher dispersion of $S$ stars and supernovae at late stages (using image tube techniques) will help to bridge the gap mentioned by Tayler.

Mustelı In a low mass supernova of $\sim 1 M_{\odot}$, only about $0.1 M_{\odot}$ is ejected and the metals probably stay inside.

Hesser to Arnett and Ostriker: Can you suggest any additional observations that will help in your quest for a satisfactory theoretical model? (This question was asked by Hesser more than once in the Symposium but never produced a reply.)

Schwarzschild: May I ask two questions? First, is the radius of the shell responsible for producing the absorption spectrum which you, Dr Mustel, have discussed in agreement with the extent of the envelope which the theoreticians seem to need to produce the characteristic sharp peak in the observed light curve? Second, could it be that the shell responsible for producing the absorption spectrum is only a small portion of the whole ejected envelope?

Woolf: Dr Mustel's star had a radius at maximum light of $2 \times 10^{15} \mathrm{~cm}$. Dr Ostriker required an envelope of size $5 \times 10^{15} \mathrm{~cm}$. The agreement is therefore excellent.

Arnett: In my model, I used a size of $1 \times 10^{15} \mathrm{~cm}$. These numbers are in agreement to within their uncertainties.

Mustel: I calculated the size of a supernova at light maximum in two different ways. I obtained $40000 R_{\odot}$ from the expansion velocity and $20000 R_{\odot}$ from the luminosity and effective temperature.

I have one further comment. There are some interesting condensations of nitrogen around supernovae which are visible during the explosion but it seems necessary to assume that they existed before the event. 